\title{
A Game Theoretic Approach to Coordinate Pricing and Advertising in Closed-loop Supply Chain
}

\author{
Jian Tan* \\ School of Management Science and Project Management, Guizhou University of \\ Finance and Economics, Guizhou 550025, China \\ tanjian123@whu.edu.cn
}

\begin{abstract}
As the main member of closed-loop supply chain, the enterprises always consider optimizing supply chain from the angles of pricing and channel while ignoring the role of advertising in the supply chain. In order to study pricing decision and advertising coordinate strategy, we divided the closed-loop supply chain into four cases including manufacture recycling advertising, retailer recycling advertising, manufacture recycling retailer advertising and manufacture advertising retailer recycling. With the impact of advertising for market demand and recycling, by using the game theory, we established four mathematical models, the results show that the retailer recycling advertising mode is the most advantageous for the closed-loop supply chain, compared with the other three modes, we found that when retailer responsible for recycling and advertising, thought the advertisement costs is highest, but the demand, profits of retailer and manufacture, recycling rate are also the highest.
\end{abstract}

Keywords: advertising, closed-loop supply chain, game theory, coordination

\section{Introduction}

Available resources on the earth are quite limited, the effective implementation of the closed-loop supply chain, especially recycling waste products, can not only realize effective resource reuse, but also can reduce environmental pollution and save the production cost. As the main member of closed-loop supply chain, the enterprises always consider optimizing supply chain from the angles of pricing and channel while ignoring the role of advertising in the supply chain. Nowadays advertisement play the same important role as price and channel. For example, scrapped automobiles can be recycled and used again, and the aim of automobile advertisement is to propaganda their product usage, product quality, and display corporate image, by this way, the manufacture can raise the vehicle sales, consumers can rely on the advertisement to guide their purchase behavior. If the automobile advertisement implicit the information about scrapped automobiles can be recycled, it not only can improve the recycling rate and saving resources as well as greatly reduce the production cost and price, but also can convey the green idea to establish a good corporate image.

However, though the advertisement will promote sales, but it will increase product cost which lead to the other problems, such as who should bear the advertisement costs in the closed-loop supply chain, what form to bear. For the sales revenue, product recycling costs and advertisement costs, how to balance between these costs and profits. Second, as we all know, reasonable profit allocation is one important condition to ensure the stability of the closed-loop supply chain, so What is the reasonable allocation and which form to allocate are also important problems. Finally, who is responsible for the recycling, who is responsible for advertising, the 
different mode of cooperation will lead to different advertising costs and profits. This article will around these issues to conduct in-depth research.

Many scholars have researched on maximum benefits for both manufacturer and retailer about supply chain coordination (Jeuland (1983), Moorthy (1987), Ingene (1995)), but most of them focus on pricing while pay less attention to pricing and advertising for supply chain coordination. Doraiswamy (1979) has studied on advertising strategy of the supply chain under the unit product fixed discount. Bergen (1997) has analyzed the supply chain advertising cooperative strategies while the retailer decides the proportion of advertisement cost. Corstjens and Lal (1989) considered supply chain cooperative advertising strategies, in the model, the retailer determines the total cost of the proportion of advertising. Jorgensen(2000) considered that advertising cost share of supply chain can be divided into four types(short-term share, long-term share, both short-term and long-term share, both short-term and long-term don't share), their research have shown that advertising cost share is benefit for manufacture, on the basis of this research, Yue (2006)obtained optimal advertising share proportion, retail price and marketing cost. Savaskan and Bhattacharya (2004) analyzed one manufacturer and one retailer closed-loop supply chain system, analyzed optimal decision of closed-loop supply chain members in different recycling channels, by compared the recycling channel retail price, wholesale price, recycling rate and the channel profit to evaluate the merits of each channel. Xie and Ai (2006) studied a game-theoretic model for cooperative advertising in a supply chain consisting of one manufacturer and one retailer. SeyedEsfahani and Biazaran (2011) considers vertical co-op advertising along with pricing decisions in a supply chain, four game-theoretic models are established in order to study the effect of supply chain power balance on the optimal decisions of supply chain members. Yue and Austin (2013) use a game theoretical approach to study pricing and advertisement decisions in a manufacturer-retailer supply chain when price discounts are offered by both the manufacturer and retailer. In Zhang and Gou (2013) model, both the consumer's goodwill and reference price for the product are assumed to be influenced by the advertising and are modeled in differential dynamic equations. Zhang and $\mathrm{Li}$ (2014) assume that both the manufacturer and the retailer can choose to participate in the advertising initiative by reducing their advertising levels, the research show that the effectiveness of the advertising initiative critically depends on the leader's participation in the initiative.

From these references can be seen, some researches just consider different recycling channel optimal decision from the price factor of closed-loop supply chain, some just study the general supply chain price and advertising investment decision problem, and there are few literature both taking into account the price and advertising factors in a closed-loop supply chain and study different recycling channel. In fact, in closed loop supply chain, advertising can not only let consumers get the product information to increase sales, but also reduce the cost and increase the market demand. It also contribute to the waste product recycling, reduce product cost.

Based on the above research foundation, take account about a closed-loop supply chain in advertising can not only influence the demand of the market, but also can affect the recycling, we will build manufacture recycling advertising model, retailer recycling advertising model, manufacture recycling retailer advertising model and manufacture advertising retailer recycling model, analyze optimal price and advertising decisions of each model, and take comparative static analysis. 


\section{Model Framework}

We consider a closed-loop supply chain with one manufacture and one retailer in which all activity occurs within a single period. The manufacturer or retailer is responsible for recycling of waste products. Because products retail price $p$ and advertisement level $m$ effect the amount of demand, suppose the demand function is $D(p, m)=\phi-\beta p+\gamma m$, which $\phi$ is the market base, $\beta$ represents the sensitive factor of demand on price, $\gamma$ is the sensitive factor of demand on advertising level. In virtue of retailer price $p$ will not less than the production cost $c_{m}$ of manufacture, while $p=c_{m}$, the amount of demand should reach the maximum, so $\phi-\beta c_{m}>0$ is permanent establishment.

There are two aspects information included by closed-loop supply chain advertisement, the one is the information for promoting the sale, and another is the information for improving waste products recovery rate. Suppose the advertising recovery satisfaction $\tau(m)=\lambda m$, which $\lambda$ represents advertising recovery efficiency factor. When they recovery waste products from consumers, the unit cost $A$ of recycling and storage of waste products must be paid to consumers. If retailer recycling of waste products, the retailer will resale them to manufacturer with unit price $b$,so $A<b$ guarantee retailer would like to recycling waste products. Suppose there are no difference between new products which produced by waste products and raw material. The unit cost of new product produced by waste product is $c_{r}$, in order to ensure manufacturer is more willing to reduce product costs by recycling waste products remanufacturing way, $c_{r}<c_{m}$ must be set up. Then $\Delta=c_{m}-c_{r}$ means unit produce cost savings by waste products, so $c_{m}-\tau \Delta$ is an average cost per unit for a new product. $A<\Delta$ must be given, so as to ensure that manufacturer willing to recycling of waste products. Set $v=\Delta-A, v$ means actual unit produce cost savings by recycling waste products. Then the total cost of closed-loop supply chain is $\kappa m^{2} / 2+A m \lambda D(p, m)$, the cost of advertising level $m$ is $m^{2} \kappa / 2, \kappa$ is strictly positive parameter we refer to as "advertisement cost factors" (G.E.Frucher.,2009).

Suppose each party is information symmetry in the closed-loop supply chain, the manufacturer has absolute leadership to the channel. Parameters $\lambda$ is small enough to ensure $\tau<1$ and $(\gamma+\beta \lambda v)^{2}-2 \beta \kappa<0$, which Guarantee if all the waste product recycling, the cost will be very large. Let $M$ represents manufacturer, $R$ represents the retailer, $T$ represents the whole supply chain. $\Pi_{l}^{i j}$ represents $l$ 's profits while $i$

recycling and $j$ advertising, which $i=j=M, R, l=M, R, T$.

The process of game for manufacturer and retailer are as follows: firstly, the manufacturer make decision; secondly, the retailer make decision. This game is a complete information dynamic game and the sub game perfect Nash equilibrium, can be solved by backward induction method.

\section{The Model}

\subsection{Manufacturer Recovery and Advertising Model (MM model)}

In this model, manufacturer responsible for recycling of waste products and advertising, retailer is responsible for product sales. 
Given wholesale price $w$ and advertising level $m$ by manufacturer, the retailer's problem is:

$$
\max _{p>0} \Pi_{{ }_{R}}^{M u}(p)=(p-w)(\phi-\beta p+\gamma m)
$$

For the given $p^{M{ }^{M}}$ by retailer due to formula (1), the manufacturer's problem at first stage is:

$$
\max _{w>0, m>0} \prod_{M}^{M M}(w, m)=\left(\phi-\beta p^{M M^{*}}+\gamma m\right)\left(p^{M M^{*}}-c_{m}+\Delta m \lambda\right)-1 / 2 \kappa m^{2}-A m \lambda\left(\phi-\beta p^{M M^{*}}+\gamma m\right)
$$

\subsection{Manufacturer Recovery and Retailer Advertising Model (MR Model)}

In this model, manufacturer responsible for recycling of waste products, retailer is responsible for product sales and advertising.

Given wholesale price $w$ by manufacturer, the retailer's problem is:

$$
\max _{p, m>0} \Pi_{R}^{M R}(p, m)=(p-w)(\phi-\beta p+\gamma m)-\kappa m^{2} / 2
$$

For the given $p^{M R^{*}}, m^{M R^{*}}$ by retailer due to formula (3), the manufacturer's problem at first stage is:

$$
\max _{w>0} \Pi_{M}^{M R}(w)=(\phi-\beta p+\gamma m)\left(w-c_{m}+\Delta m \lambda\right)-A m \lambda(\phi-\beta p+\gamma m)
$$

\subsection{Retailer Recovery and Advertising Model (RR Model)}

In this model, manufacturer just responsible for produce products, retailer is responsible for product sales, advertising and recovery.

Given wholesale price $w$ by manufacturer, the retailer's problem is:

$$
\max _{p>0, m>0} \prod_{R}^{R R}=(p-w)(\phi-\beta p+\gamma m)+b \lambda m(\phi-\beta p+\gamma m)-\kappa m^{2} / 2-A \lambda m(\phi-\beta p+\gamma m)
$$

For the given $p^{R^{*}}, m^{R R^{*}}$ by retailer due to formula (5), the manufacturer's problem at first stage is:

$$
\max _{w>0} \Pi_{M}^{R R}=\left(w-c_{m}+\Delta m \lambda\right)(\phi-\beta p+\gamma m)-b \lambda m(\phi-\beta p+\gamma m)
$$

\subsection{Retailer Recovery and Manufacturer Advertising Model (RM Model)}

In this model, manufacturer responsible for advertising, the retailer is responsible for product sales and recycling of waste products.

Given wholesale price $w$ and advertising level $m$ by manufacturer, the retailer's problem is:

$$
\max _{p>0} \Pi_{R}^{R M}(p)=(p-w)(\phi-\beta p+\gamma m)+b \lambda m(\phi-\beta p+\gamma m)-A \lambda m(\phi-\beta p+\gamma m)
$$

For the given $p^{R M *}$ by retailer due to formula (7), the manufacturer's problem at first stage is:

$$
\max _{m>0, w>0} \Pi_{M}^{R M}=\left(w-c_{m}+\Delta m \lambda\right)(\phi-\beta p+\gamma m)-b \lambda m(\phi-\beta p+\gamma m)-\kappa m^{2} / 2
$$




\section{Comparative Analysis of Advertising Decision Models}

According to the above four models, using backward induction method, obtained the results as shown in Table 1.

Proposition 1: While $\gamma / \lambda \geq \beta v, w^{R M^{*}}>w^{M M^{*}} \geq w^{R R^{*}}>w^{M R^{*}}$; if else, $w^{R M^{*}}>w^{R R^{*}}>w^{M M^{*}}>w^{M R^{*}}$.

Proof : From table 1, we get

$$
\begin{aligned}
& w^{M M^{*}}-w^{R M^{*}}=\frac{-v E(\gamma+\beta \lambda v)}{C}<0, w^{R R^{*}}-w^{R M^{*}}=-\frac{(\gamma+\beta \lambda v)^{2} E}{2 \beta C}<0, \\
& w^{M R^{*}}-w^{M M^{*}}=-\frac{E\left(\gamma^{2}(C-2 \beta \kappa)+4 \beta^{2} \gamma \kappa \lambda v\right)}{2 \mathrm{~B} C}<0, w^{R R^{*}}-w^{M R^{*}}=\frac{v \kappa \lambda E}{2 B}>0 .
\end{aligned}
$$

Because $w^{M M^{*}}-w^{R R^{*}}=\frac{(\beta \lambda v-\gamma)(\beta \lambda v+\gamma) E}{2 \beta C}$, hence, while $\gamma / \lambda \geq \beta v, w^{M M^{*}} \geq w^{R R^{*}}$; while $\gamma / \lambda<\beta v, w^{M M^{*}}<w^{R R^{*}}$.

Proposition 2: While $\gamma / \lambda \geq \beta V, p^{R M *}=p^{M M^{*}}>p^{R R^{*}} \geq p^{M R^{*}}$; if else,

$$
p^{R M^{*}}=p^{M M^{*}}>p^{M R^{*}}>p^{R R^{*}} .
$$

Proof: From table 1, we can get $p^{R M^{*}}=p^{M M^{*}}$ directly. Because $p^{R R^{*}}-p^{M R^{*}}=\frac{\beta v \kappa \lambda E(\gamma-\beta \lambda v)}{2 B(C-2 \beta \kappa)}$, so when $\gamma / \beta \geq \lambda v, p^{R R^{*}} \geq p^{M R^{*}}$;

$$
\begin{aligned}
& \text { when } \gamma / \beta \leq \lambda v, p^{R R^{*}} \leq p^{M R^{*}} \cdot p^{R R^{*}}-p^{R M^{*}}=-\frac{(\gamma+\beta \lambda v)^{2}(B+2 \beta \gamma v) E}{2 \beta B C}<0, \\
& p^{M R^{*}}-p^{M M^{*}}=-\frac{E\left(\left(\gamma^{2}+\beta \kappa\right)(C-2 \beta \kappa)+4 \beta^{2} \gamma \kappa \lambda v\right)}{2 B C}<0 .
\end{aligned}
$$

Proposition 3: $m^{R R^{*}}>m^{M M *}=m^{R M *}>m^{M R^{*}}$.

Proof: From table 1, we can get $m^{M M^{*}}=m^{\mathrm{RM} *}$ directly. $m^{R R^{*}}-m^{R M *}=\frac{(\gamma+\beta \lambda v)^{3} E}{2 C(C-2 \beta \kappa)}>0, m^{R R^{*}}-m^{M R^{*}}=\frac{\beta^{2} v \kappa \lambda E}{B(C-2 \beta \kappa)}>0$,

$$
m^{R R^{*}}-m^{R M *}=\frac{(\gamma+\beta \lambda v)^{3} E}{2 C(C-2 \beta \kappa)}>0 .
$$

Which $B=-\gamma^{2}+2 \beta \kappa-\beta \gamma \lambda v, E=\phi-\beta c_{m}, C=-(\gamma+\beta \lambda v)^{2}+4 \beta \kappa, F=\phi+\beta c_{m}$.

Proposition 4: While $\quad \gamma / \lambda \geq \beta V \quad, \quad D^{R R^{*}}>D^{M R^{*}} \geq D^{M M^{*}}=D^{R M^{*}} \quad ; \quad$ if else, $D^{R R^{*}}>D^{M M^{*}}=D^{R M^{*}} \geq D^{M R^{*}}$.

Proof: From Table 1, we can get $D^{M M^{*}}=D^{R M^{*}}$ directly. Because $D^{M R^{*}}-D^{M M^{*}}=\frac{\beta \kappa E(\gamma+\beta \lambda v)(\gamma-\beta \lambda v)}{2 B C}$, so while $\gamma / \lambda \geq \beta v, D^{M R^{*}} \geq D^{M M^{*}}$; 
while $\quad \gamma / \lambda<\beta v \quad, \quad D^{M R^{*}}<D^{M M^{*}} \quad . \quad D^{R R^{*}}-D^{M M^{*}}=\frac{\beta \kappa E(\gamma-\beta \lambda v)^{2}}{2(C-2 \beta \kappa) C}>0$, $D^{R R^{*}}-D^{R M^{*}}=\frac{\beta \kappa(C+2 \beta \kappa) E}{2 C(C-2 \beta \kappa)}>0, D^{R R^{*}}-D^{M R^{*}}=\frac{\kappa \beta^{2} \lambda\left(v \gamma+\beta \lambda v^{2}\right) E}{2 B(C-2 \beta \kappa)}>0$.

Proposition 5:

$$
\begin{aligned}
& \Pi_{R}^{R R^{*}}>\max \left(\Pi_{R}^{M M^{*}}, \Pi_{R}^{R M *}, \Pi_{R}^{M R^{*}}\right), \quad \Pi_{M}^{R R^{*}}>\max \left(\Pi_{M}^{M R^{*}}, \Pi_{M}^{M M^{*}}, \Pi_{M}^{R M^{*}}\right), \\
& \Pi_{T}^{R R^{*}}>\max \left(\Pi_{T}^{M M *}, \Pi_{T}^{R M^{*}}, \Pi_{T}^{M R^{*}}\right) . \\
& \text { Proof: } \prod_{R}^{M M^{*}}-\Pi_{R}^{R R^{*}}=-\frac{\kappa E^{2}(\gamma+\beta \lambda v)^{4}}{8 C(C-2 \beta \kappa)}<0, \Pi_{R}^{R R^{*}}-\Pi_{R}^{R M *}=\frac{\kappa(\gamma+\beta \lambda v)^{4} E^{2}}{8 C^{2}(C-2 \beta \kappa)}>0, \\
& \Pi_{R}^{R R^{*}}-\Pi_{R}^{M R^{*}}=\frac{\kappa^{2} \beta^{3} \lambda^{2} v^{2} E}{4 B^{2}(C-2 \beta \kappa)}>0 \text {, so we get } \Pi_{R}^{R R^{*}}>\max \left(\prod_{R}^{M M^{*}}, \Pi_{R}^{R M^{*}}, \Pi_{R}^{M R^{*}}\right) \text {. } \\
& \text { Hence } \Pi_{M}^{M M^{*}}=\Pi_{M}^{R M^{*}}, \Pi_{M}^{R R^{*}}-\Pi_{M}^{M M^{*}}=\frac{\kappa E^{2}(\gamma-\beta \lambda v)^{2}}{4(C-2 \beta \kappa) C}>0 \text {, } \\
& \Pi_{M}^{R R^{*}}-\Pi_{M}^{M R^{*}}=\frac{\kappa \beta \lambda\left(v \gamma+\beta \lambda v^{2}\right) E}{4 B(C-2 \beta \kappa)}>0 \text {, so } \Pi_{M}^{R R^{*}}>\max \left(\Pi_{M}^{M R^{*}}, \Pi_{M}^{M M^{*}}, \Pi_{M}^{R M^{*}}\right) . \mathrm{Th}
\end{aligned}
$$

en we have $\Pi_{T}^{R R^{*}}>\max \left(\Pi_{T}^{M M^{*}}, \Pi_{T}^{R M *}, \Pi_{T}^{M R^{*}}\right)$.

Proposition 6: In the above four models, if the advertising recovery efficiency factor or advertising demand efficiency factor increases, 1) the optimal advertising level increases; 2) the market demand increases; 3) manufacturer, retailer, the overall profits increase.

Proof: By first order derivative about $m, D, \Pi_{M}, \Pi_{R}$ and $\Pi_{T}$ with $\gamma$ and $\lambda$ respectively, all of them are greater than 0 .

Proposition 6 shows that the advertising recovery efficiency factor and advertising demand efficiency factor are more higher, the advertising level would be higher, which promote market demand and waste product rate higher, then the average product cost reduced, and ultimately improve profits of the closed-loop supply chain.

\section{Numerical Examples}

In order to verify the models, we set $\phi=10000, c=10, \gamma=5, \Delta=7, \lambda=0.003$, $A=4, \kappa=1$. The results of simulation are as shown in table 2 -table 3 and figure 1 figure 2 . 
Table 1. The Results of the Models

\begin{tabular}{|c|c|c|c|c|}
\hline & $M R$ & $M M$ & $R R$ & $R M$ \\
\hline \multirow{3}{*}{$p$} & $\phi B+\kappa F \beta$ & $(3 \kappa+v \lambda(-\gamma-\lambda \beta v)) \phi$ & $\phi C+(\kappa-v \gamma \lambda) F \beta$ & $(3 \kappa+v \lambda(-\gamma-\lambda \beta v)) \phi$ \\
\hline & $-\gamma \lambda v \phi \beta-\gamma^{2} \beta c_{m}$ & $+(\mathrm{B}-\beta \kappa+2 \beta \gamma v \lambda) c_{m}$ & $-\beta^{2} \lambda^{2} v^{2} \phi-\beta^{2} \gamma^{2} c_{m}$ & $+(B-\beta \kappa+2 \beta \gamma \nu \lambda) c_{m}$ \\
\hline & $2 B \beta$ & $C$ & $2 C \beta$ & $C$ \\
\hline \multirow{3}{*}{$w$} & & $2 \kappa F-\lambda \gamma \nu F$ & $F$ & $\mathrm{FC}+(4 \beta \kappa-C)^{2} E$ \\
\hline & $+\beta c_{m}(B+\beta \gamma \lambda v)$ & $-\beta \lambda^{2} v^{2} \phi+\gamma^{2} c_{m}$ & $\overline{2 \beta}$ & $2 \beta C$ \\
\hline & $2 B \beta$ & $C$ & & \\
\hline \multirow{2}{*}{$m$} & $\underline{\gamma E}$ & $(\gamma+\beta \lambda v) E$ & $\underline{(\gamma+\beta \lambda v) E}$ & $(\gamma+\beta \lambda v) E$ \\
\hline & $2 B$ & C & $2 C$ & $C$ \\
\hline \multirow{2}{*}{$D$} & $\beta \kappa E$ & $\beta \kappa E$ & $\beta \kappa E$ & $\beta \kappa E$ \\
\hline & $2 B$ & $C$ & $2 C-4 \beta \kappa$ & $C$ \\
\hline \multirow{2}{*}{$\Pi_{R}$} & $\kappa(B+\beta \gamma \lambda v) E^{2}$ & $\beta \kappa^{2} E^{2}$ & $\kappa E^{2}$ & $\beta \kappa^{2} E^{2}$ \\
\hline & $8 B^{2}$ & $C^{2}$ & $8 C-16 \beta \kappa$ & $C^{2}$ \\
\hline \multirow{2}{*}{$\Pi_{M}$} & $\kappa E^{2}$ & $\kappa E^{2}$ & $\kappa E^{2}$ & $\kappa E^{2}$ \\
\hline & $4 B$ & $2 C$ & $4 C-8 \beta \kappa$ & $2 C$ \\
\hline \multirow{2}{*}{$\Pi_{T}$} & $\kappa(3 \mathrm{~B}+\beta \gamma \lambda v) E^{2}$ & $\kappa(C+2 \beta \kappa) E^{2}$ & $3 \kappa E^{2}$ & $\kappa(C+2 \beta \kappa) E^{2}$ \\
\hline & $8 B^{2}$ & $2 C^{2}$ & $8 C-16 \beta \kappa$ & $2 C^{2}$ \\
\hline
\end{tabular}

Table 2. The Simulation while ${ }^{\gamma / \lambda>\beta v}$ which $^{\beta}=100$

\begin{tabular}{ccccc}
\hline & MR & MM & RR & RM \\
\hline$w$ & 53.81 & 57.98 & 55.00 & 59.29 \\
$p$ & 80.21 & 82.63 & 80.79 & 82.63 \\
$m$ & 132 & 145 & 161 & 145 \\
$\Pi_{M}$ & 118768 & 110901 & 122586 & 110901 \\
$\Pi_{R}$ & 60951 & 60736 & 61293 & 60736 \\
$\Pi_{T}$ & 179720 & 171637 & 183879 & 171637 \\
$D$ & 2639 & 2464 & 2724 & 2464 \\
$\tau$ & $39.59 \%$ & $43.62 \%$ & $48.22 \%$ & $43.62 \%$ \\
\hline
\end{tabular}

Table 3. The simulation while ${ }^{\gamma / \lambda<\beta v}$ which $^{\beta}=600$

\begin{tabular}{ccccc}
\hline 1 & MR & MM & RR & RM \\
\hline$w$ & 13.25 & 13.32 & 13.33 & 13.49 \\
$p$ & 15.00 & 15.07 & 14.99 & 15.07 \\
$m$ & 8.71 & 18.15 & 19.05 & 18.15 \\
$\Pi_{M}$ & 3484 & 3491 & 3664 & 3491 \\
$\Pi_{R}$ & 1783 & 1828 & 1832 & 1828 \\
$\Pi_{T}$ & 5267 & 5318 & 5495 & 5318 \\
$D$ & 1045 & 1047 & 1099 & 1047 \\
$\tau$ & $2.61 \%$ & $5.45 \%$ & $5.72 \%$ & $5.45 \%$ \\
\hline
\end{tabular}

From Table 2 and Table 3, we can get that no matter the relationship between $\gamma / \lambda$ and $\beta v$, compared to the other modes, the market demand, the advertising level, recovery rate, the profits of retailer and manufacture are highest, hence, we can conclude that the RR mode is benefit to the closed-loop supply chain. Especially, 
when $\beta=600$, the recovery rate far less than $\beta=100$, the reason is that, when the price elasticity of demand $\beta$ is large, the effect of advertisement is smaller to the market demand compared to price. Hence, the price strategy is the main way to gain market which lead to lower remanufacture profits, and the advertising level is lower, so eventually lead to recovery rate is lower.

Actually, the profits and costs coordination between the manufacturer and the retailer is mainly by the wholesale price.

From Figure 1 and Figure 2 can be seen, the wholesale price is largest in the RM mode, because the retailer recovery waste products and resell to manufacturer to obtain profits, the manufacture share the part of the profits by raising wholesale price, at the same time, the retailer should bear a part of advertisement costs of manufacture, which is reflected by the wholesale price enhance. Corresponding with RM mode is the MR model, the wholesale price is smallest in the MR mode because the manufacturer recovery waste products to save product cost, the retailer share the part of saving costs by reducing wholesale price, at the same time, the manufacturer should bear a part of advertisement costs of retailer, which is reflected by the wholesale price reduce. For the RR mode which retailer recovery and advertising, the wholesale price has unrelated to with advertising recovery efficiency factor and advertising marketing efficiency factor, because the profits obtained though recovery waste products and resold to manufacturer can offset the costs of advertising.

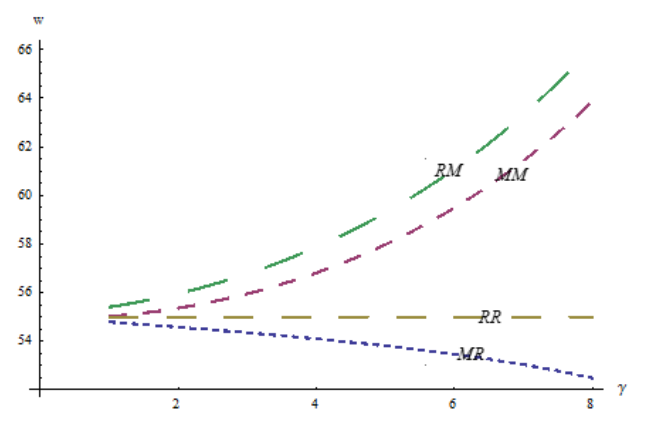

Figure 1. $w$ changes with $\gamma$

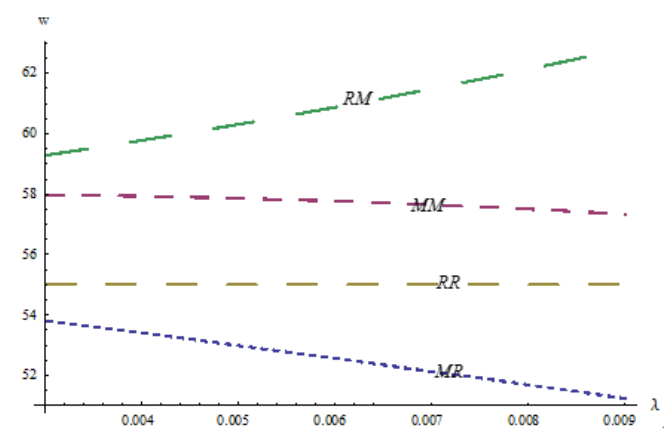

Figure 2. $w$ changes with $\lambda$

We note that the wholesale price decrease as advertising marketing efficiency factor and recovery efficiency factor increase in the MR mode. Because the manufacture recovery waste products can reduce product cost while the retailer should pay the advertisement costs, when the advertising marketing efficiency factor or recovery efficiency factor increase, the demand, advertising level and the profits of manufacture all increase, the manufacturer should be reducing the wholesale price to compensate the retailer's advertising costs and profits sharing at the same time. 


\section{Conclusion}

In this paper, In order to study pricing decision and advertising coordinate strategy, we divided the closed-loop supply chain into four cases including manufacture recycling advertising, retailer recycling advertising, manufacture recycling retailer advertising and manufacture advertising retailer recycling. With the impact of advertising for market demand and recycling, by using the game theory, we established four mathematical models, the results show that the retailer recycling advertising mode is the most advantageous for the closed-loop supply chain, compared with the other three modes, we found that when retailer responsible for recycling and advertising, thought the advertisement costs is highest, but the demand, profits of retailer and manufacture, recycling rate are also the highest.

\section{Acknowledgments}

This work is supported by the science and technology foundation of Guizhou province (Guizhou J word [2013]2085), Guizhou Natural Science Foundation Project ((2014) 264).

\section{References}

[1] A. A. Javid and P. Hoseinpour, "A cooperative advertising model for a supply chain with one manufacturer and one retailer", European Journal of Operational Research, vol. 219, no. 2, (2012), pp. 458-466.

[2] M. Bergen and G. John, "Understanding Cooperative Advertising Participation Rates in Conventional Channels", Journal of Marketing Research, vol. 8, no. 46, (1997), pp. 357-369.

[3] M. Corstjens and R. Lal, "Cooperative Advertising and Channel Coordination", ResearchPaper\#1071, Graduate School of Business, Stanford University, (1989).

[4] K. Doraiswamy, T. W. McGuire and R. Staelin, "An Analysis of Alternative Advertising Strategies Competitive Franchise Framework", Educators' Conference Proceedings, American Marketing Association, (1979).

[5] G. E. Frucher, "Signaling Quality: Dynamic price-Advertising Model”, Journal of Optimization Theory and Applications, vol. 143, no. 3, (2009), pp. 479-496.

[6] C. A. Ingene and M. E. Parry, "Channel Coordination when Retailers Compete", Marketing Science, vol. 14, no. 4, (1995), pp. 360-377.

[7] C. A. Ingene and M. E. Parry, "Coordination and Manufacturer Profit Maximization: The Multiple Retailer Channel”, Journal of Retailing, vol. 35, no. 2, (1995), pp. 129-151.

[8] A. P. Jeuland and S. M. Shugan, "Managing Channel Profits", Marketing Science, vol. 27, no. 1, (2008), pp. 52-69.

[9] S. Jorgensen and G. Zaccour, "Dynamic cooperative advertising in a channel", Journal of Retailing, vol. 76, no. 1, (2000), pp. 71-92.

[10] K. S. Moorthy, "Managing Channel Profits: Comment", Marketing Science, vol. 6, no. 4, (1987), pp. 375379.

[11] Z. Pei, L. Toombs and R. Yan, "How does the added new online channel impact the supporting advertising expenditure?", Journal of Retailing and Consumer Services, vol. 21, no. 3, (2014), pp. 229238.

[12] R. Savaskan, C. S. Bhattacharya and L. Van Wasaenhove, "Closed-loop supply chain models with product remanufacturing”, Management Science, vol. 50, no. 2, (2004), pp. 239-253.

[13] J. Xie and S. Ai, "A note on cooperative advertising, game theory and manufacturer-retailer supply chains", Omega, vol. 34, no. 5, (2006), pp. 501-504.

[14] M. M. SeyedEsfahani, M. Biazaran, M. Gharakhani, "A game theoretic approach to coordinate pricing and vertical co-op advertising in manufacturer-retailer supply chains", European Journal of Operational Research, vol. 211, no. 2, (2011), pp. 263-273.

[15] J. Yue, J. Austin and Z. Huang, "Pricing and advertisement in a manufacturer-retailer supply chain", European Journal of Operational Research, vol. 231, no. 2, (2013), pp. 492-502.

[16] J. Yue, "Coordination of cooperative advertising in a two-level supply chain when manufacturer offers discount", European Journal of Operational Research, vol. 168, no. 1, (2006), pp. 65-68.

[17] J. Zhang, Q. Gou and L. Liang, "Supply chain coordination through cooperative advertising with reference price effect", Omega, vol. 41, no. 2, (2013), pp. 345-353.

[18] W. Zhang, S. Li and D. Zhang, "The impact of advertising initiatives in supply chains", European Journal of Operational Research, vol. 234, no. 1, (2014), pp. 99-107. 
International Journal of $u-$ and e- Service, Science and Technology

Vol.8, No. 11 (2015)

\section{Author}

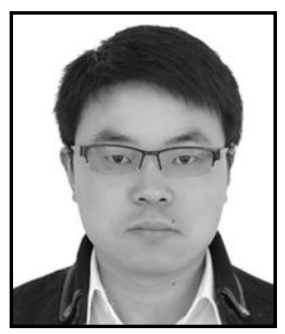

Jian Tan, 1982-, male, Hubei Province of China, Ph. D, associate professor, research direction: game theory, supply chain management. 